\title{
Fabrication of luminescent nanostructures by electron-beam direct writing of PMMA resist
}

\author{
C.A. Barrios ，S. Carrasco ，V. Canalejas-Tejero , D. López-Romero , F. Navarro-Villoslada ， \\ M.C. Moreno-Bondi , J.L.G. Fierro , M.C. Capel-Sánchez
}

\begin{abstract}
A B S T R A C T
We report on the conversion of non-luminescent conventional poly(methylmethacrylate) (PMMA)based electron-beam resists into luminescent materials when used as negative-tone resists, that is, when exposed to high electron irradiation doses. Raman spectroscopy reveals the chemical transformation induced by electron irradiation which is responsible for the observed luminescence in the visible (blue) region. The emission intensity from exposed PMMA-based patterns can be controlled by the electron irradiation dose employed to create them.
\end{abstract}

\section{Introduction}

Uses of luminescent nanostructures for photonic, chemical, and biological applications are numerous. Fluorescent nanoparticles are widely employed for biological assays [1] and chemical sensors [2]; light-emitting nanowires are appealing elements for implementing novel nanophotonic devices; and luminescent nanofilms are being developed as flexible functional coating materials for security, labeling [3] and sensing purposes [4].

Non-emissive (passive) materials can be used to create luminescent nanostructures by incorporating fluorescent additives (dopants), such as dyes and quantum dots, and subsequent nanopatterning, demanding at least two fabrication steps. A different approach to produce luminescent organic nanoarchitectures, requiring just one processing step, was demonstrated by Lee et al. [5]. These authors used electron-beam direct writing lithography (EBL) in a polystyrene (PS) film to convert the initially non-luminescent PS material into a luminescent organic material, while patterning the polymer to form nanostructures.

EBL is the most popular nanolithography technique and poly(methylmethacrylate) (PMMA) and its derivatives, such as PMMA-methacrylic acid (MA) copolymer, are the most widely used polymeric EBL resists due to their high resolution, stability, excellent adhesion to substrates, and compatibility with other processing steps. In most applications, PMMA-based resists are used as positive (i.e., the irradiated regions are developed) resists, requiring low electron dose $\left(80-100 \mu \mathrm{C} / \mathrm{cm}^{2}\right)$ to be removed. However, it is well known that PMMA can be transformed into a negative resist (i.e., the irradiated regions are retained) at high electron dose, offering ultrahigh-resolution (sub-10 nm half pitch) [6]. This behavior, due to its simple realization, has applications in making masks [7], mechanical building blocks [8], and dielectric layers or gaps [9].

In this paper, we describe and study a novel use of (negative) PMMA based resists: the straightforward (one-step) fabrication of luminescent organic nanopatterns by EBL. Raman spectroscopy is used to analyze the effect of electron irradiation and its relation with the observed photoluminescence (PL). Three aspects make this work particularly relevant as compared to that of PS [5]: (i) PMMA has no aromatic rings (unlike PS), which makes its transformation into a luminescent material particularly remarkable; (ii) the observation of this effect on the most used polymeric EBL resist (the use of polystyrene as an e-beam resist is incidental) extends its potential applications and integration capabilities significantly; and (iii) the dualbehavior of PMMA allows technologists to design and fabricate a wide range of nanostructures by controlling only the writing electronic dose.

\section{Experimental}

PMMA (950k molecular weight, in chlorobenzene) and PMMA/MA (in 2-methoxy-ethanol) from Allresist GmbH (Am Biotop 14, 15344 
Strausberg, Germany) were used for EBL exposures. (500 \pm 10$) \mathrm{nm}$ thick PMMA and $(300 \pm 10) \mathrm{nm}$ thick PMMA/MA resist layers were spin-coated on Si substrates. After spin-coating, the samples were baked on a hot plate at $160^{\circ} \mathrm{C}$ for $2 \mathrm{~min}$. Then, arrays of $50 \times 50 \mu \mathrm{m}^{2}$ solid squares with dose ranging from 7 to $25 \mathrm{mC} / \mathrm{cm}^{2}$ and line grids (pitch $=500 \mathrm{~nm}$ ) with dose ranging from 100 to $200 \mathrm{nC} / \mathrm{cm}$ were written by a CRESTEC CABL-9500C EBL system (accelerating voltage $=50 \mathrm{kV}$ and beam current $=1 \mathrm{nA}$ ). After EBL exposure, the samples were developed in IPA:MIBK (AR 600-55 from Allresist $\mathrm{GmbH}$ ) at room temperature for $2 \mathrm{~min}$, rinsed in IPA and dried with $\mathrm{N}_{2}$. The height of the solid squares after development was measured by a surface profiler (KLA Tencor Alpha-Step IQ).

The surface morphology of the grids was characterized by an atomic force microscope (AFM) (Digital Instruments MultiMode Scanning Probe Microscope Model MMAFM-2) and a Leica Leitz DMRX optical microscope.

PL images were measured at room temperature by a confocal fluorescence microscope (Olympus X51, Japan). A Hg-Xe lamp was used as the excitation source with a $405 \mathrm{~nm}$ interferential excitation filter, a $414 \mathrm{~nm}$ dichroic mirror and a $425 \mathrm{~nm}$ cut-off filter. The microscope was coupled to a FluoroMax 4 (Horiba Scientific, NJ USA) spectrofluorometer to collect the emission spectra (slit width $8 \mathrm{~nm}$ ) of the exposed resist patterns.

Raman spectra were recorded at room temperature with a Renishaw in Via Raman Microscope spectrometer equipped with a laser beam emitting at $532 \mathrm{~nm}$ and $5 \mathrm{~mW}$ output power. The photons scattered by the sample were dispersed by a 1800 lines/mm grating monochromator and simultaneously collected on a CCD camera; the collection optics was set at $50 \times$ objective. Accurate calibration was carried out by checking the Rayleigh band and Si band at 0 and $520.7 \mathrm{~cm}^{-1}$, respectively.

\section{Results and discussion}

Figs. 1a and b show bright field optical microscope images of PMMA and PMMA/MA squares, respectively, exposed to various electron irradiation doses. EBL proximity effect is clearly observed: trenches around the squares were removed (positive resist behavior) due to the scattered low electronic dose. The thickness of the squares as a function of electron irradiation dose (i.e., contrast curve) is depicted in Fig. 2. Resist contrast $(\gamma)$ is defined as $\gamma=\left[\log \left(D_{100} / D_{0}\right)\right]^{-1}$, where $D_{0}$ (threshold dose) and $D_{100}$ are the intersection values of the line with the highest slope with the zero and full resist thickness lines, respectively. The contrast values for PMMA and PMMA/MA were found to be $\gamma_{\mathrm{PMMA}}=4.4$ and $\gamma_{\text {PMMA/MA }}=3.1$, whereas the threshold doses for PMMA and a

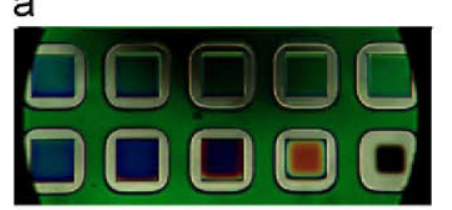

c

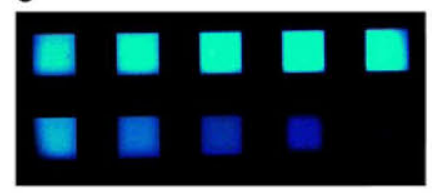

b

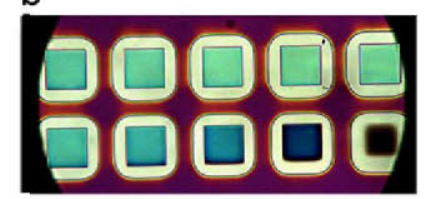

d

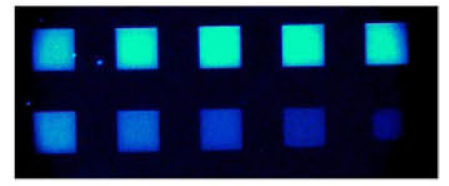

Fig. 1. Optical microscope photographs of arrays of $50 \times 50 \mu \mathrm{m}^{2}$ PMMA (a) and PMMA/MA (b) resist squares irradiated with various electron doses. Dose increases in clockwise direction from $7 \mathrm{mC} / \mathrm{cm}^{2}$ (bottom right) to $25 \mathrm{mC} / \mathrm{cm}^{2}$ (top right) in steps of $2 \mathrm{mC} / \mathrm{cm}^{2}$. PL images of the PMMA (c) and PMMA/MA (d) squares at room temperature $\left(\lambda_{\mathrm{ex}}=405 \mathrm{~nm}\right)$.

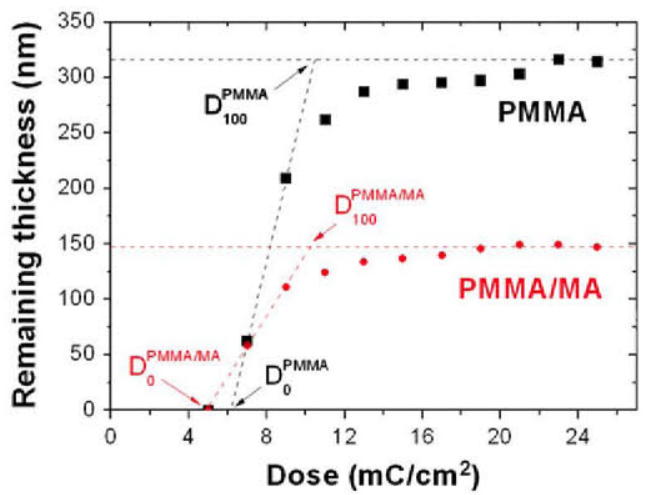

Fig. 2. Remaining thickness of the exposed PMMA (square dots) and PMMA/MA (circle dots) after development versus electron dose.
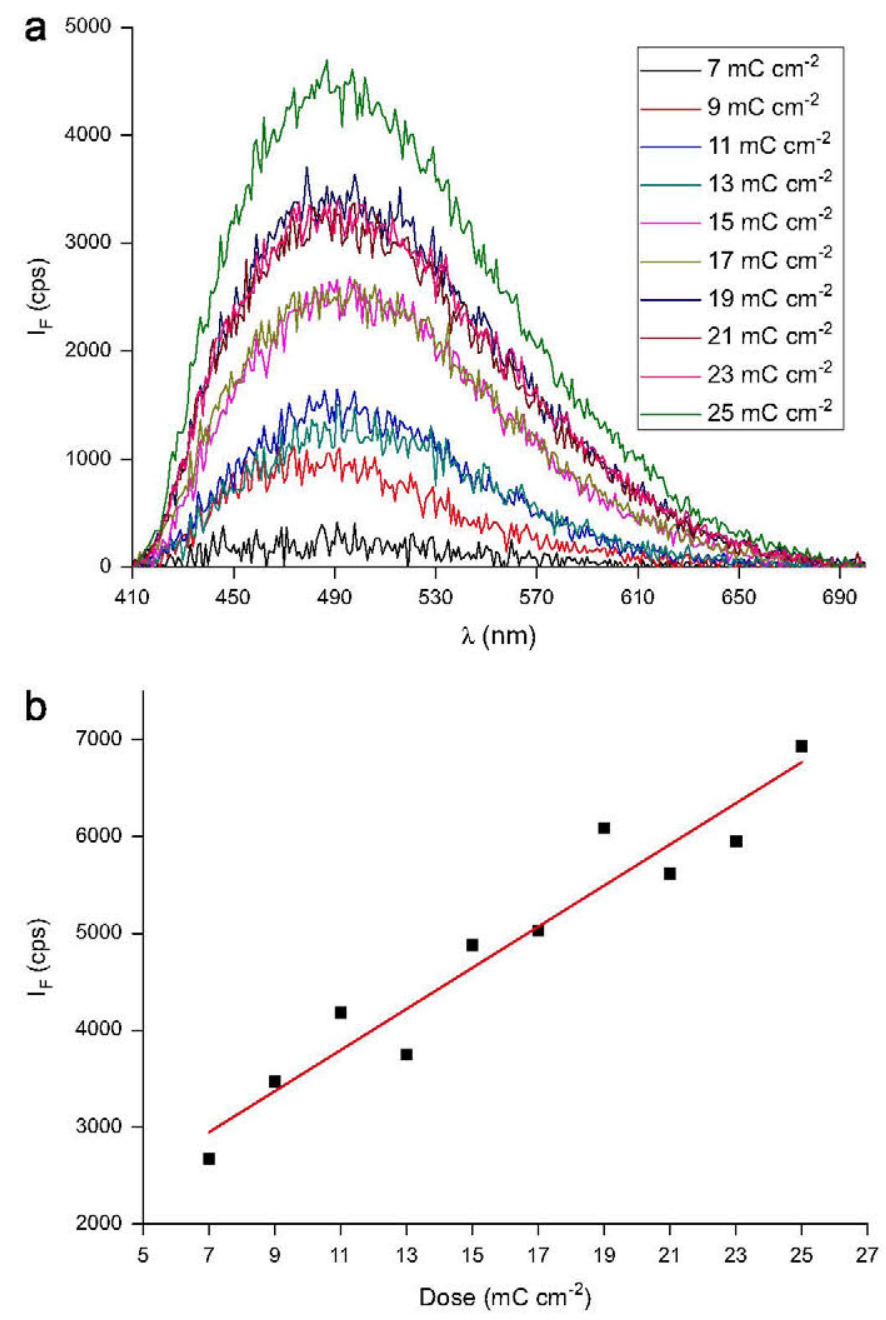

Fig. 3. (a) Photoluminescence spectra of e-beam exposed PMMA squares for several doses. (b) Variation of the emission intensity signal as a function of the electron dose.

PMMA/MA were $D_{0}^{\text {PMMA }}=6.2 \mathrm{mC} / \mathrm{cm}^{2}$ and $D_{0}^{\text {PMMA } / \mathrm{MA}}=4.9 \mathrm{mC} / \mathrm{cm}^{2}$, respectively. These values are in agreement with those reported by other authors [6].

Figs. 1c and d show fluorescence microscopy images corresponding to Figs. 1a and b, respectively. Both, PMMA and PMMA/ MA irradiated resist squares exhibit significant visible PL, while no luminescence from the unexposed resist regions is observed. Fig. 3a shows the emission spectra for the PMMA irradiated 


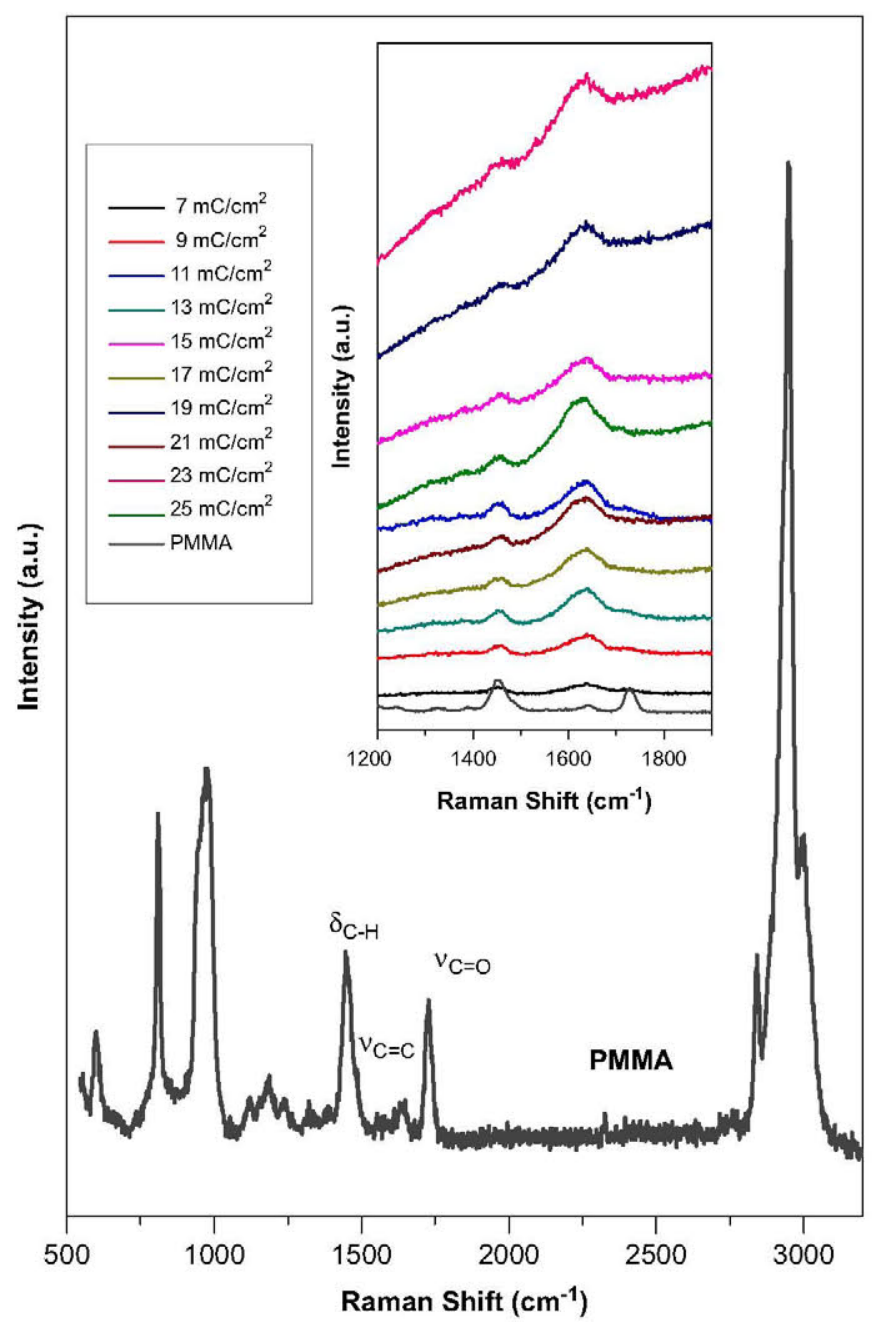

Fig. 4. Raman spectra of unexposed PMMA and e-beam exposed PMMA squares for several doses (inset).

resist squares. The PL peaks are centered at $\sim 485 \mathrm{~nm}$ and their intensities increase approximately linearly with the dose (Fig. 3b). Neither significant red-shift of the peak spectrum nor an increase of the full width at half maximum (FWHM) with the increment of the dose was observed. This is in contrast to the reported results on irradiated PS [5], which were attributed to an increment in the average cluster size of polycyclic aromatic hydrocarbons (PAH) generated by electron irradiation, suggesting a different PL origin for the PMMA patterns.

Fig. 4 shows the Raman spectra of the unexposed PMMA and exposed PMMA resist squares in the spectral region 1200$2000 \mathrm{~cm}^{-1}$. The experimental spectra contain a broad structureless background corresponding to the contribution of photoluminescence, which increases with the increment of the dose. The new band around $1636 \mathrm{~cm}^{-1}$ developed upon electron dosing confirms the presence of $\mathrm{C}=\mathrm{C}$ bonds [10]. Visible PL shown by organic materials is typically related to the existence of conjugated double bonds in the form of olefinic chains or aromatic rings. Since the dose-dependent PL spectral behavior of the irradiated PMMA resist differs from that of the irradiated PS and no aromatic $\mathrm{C}-\mathrm{H}$ stretching vibrations are detected, we attribute the origin of the $\mathrm{PL}$ of the exposed PMMA resist to the formation of olefinic chains rather than $\mathrm{PAH}$ generation.

Luminescent nanopatterns from both PMMA and PMMA/MA resists were prepared through EBL. In particular, resist grids were created by electron-beam writing of two overlapping perpendicular

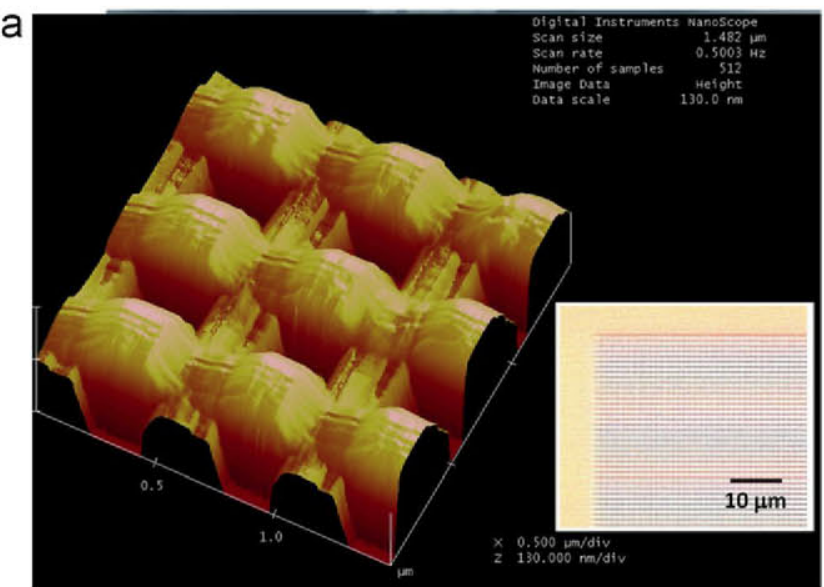

b

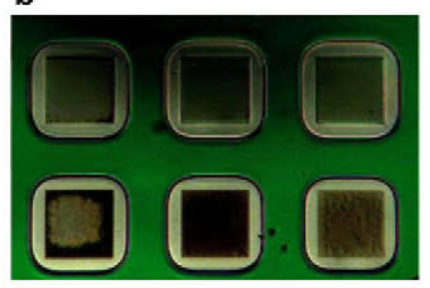

Fig. 5. (a) AFM image of a representative portion of an EBL-nanopatterned PMMA grid. Inset: microscope photograph of the 2D line grating. (b) Optical microscope photograph of an array of $50 \times 50 \mu \mathrm{m}^{2}$ PMMA resist grids produced by different electron doses. Dose increases in clockwise direction from $100 \mathrm{nC} / \mathrm{cm}$ (bottom right) to $200 \mathrm{nC} / \mathrm{cm}$ (top right) in steps of $20 \mathrm{nC} / \mathrm{cm}$. (c) PL image of the PMMA grids at room temperature $\left(\lambda_{\mathrm{ex}}=405 \mathrm{~nm}\right)$

line arrays (pitch $=500 \mathrm{~nm}$ ). Fig. 5a shows an AFM image and an optical microscope photograph (inset) of a representative portion of a PMMA grid, whereas Figs. 5b and $\mathrm{c}$ show bright field and fluorescence microscope images, respectively, of six PMMA grids created at various electronic doses. Both, PMMA and PMMA/MA nanopatterns, showed significant luminescence, their intensity being linearly correlated with the electron irradiation dose. To our knowledge, this is the first reported demonstration of PL emission from an electron-irradiated PMMA EBL resist.

We have presented PL blue emission from conventional PMMA and PMMA/MA resists when operated as negative-tone EBL resists. Raman spectroscopy measurements point to the formation of olefinic chains as the origin of the observed PL. The emitted light intensity correlates linearly with the electron irradiation dose used to create the patterns. This work demonstrates a straightforward (one-step) method to fabricate arbitrary light-emitting organic nanopatterns on chips based on the most employed nanolithography technique, EBL, and the most popular EBL resist. Potential applications of negative PMMA resist-based organic nanostructures include integration of light sources in photonic and optofluidic chips and fabrication of nanometer scale markers for fluorescent microscopy, security issues and biochemical sensing.

\section{Acknowledgments}

The authors acknowledge financial support from MICINN (TEC2010-10804-E, CTQ2009-14565-C03-03) and the Moncloa Campus of International Excellence (CEI-Moncloa).

\section{References}

[1] Han M, Gao X, Su JZ, Nie S. Nat Biotechnol 2001;19:631.

[2] Chen L, McBranch DW, Wang HL, Helgeson R, Wudi F, Whitten DG. Proc Natl Acad Sci USA 1999;96:12287. 
[3] Hiraga T, Iketaki Y, Watanabe T, Ohyi H, Kobayashi K, Yamamoto N, et al. Rev Sci Instrum 2005;76:073701.

[4] Aparicio FJ, Holgado M, Borras A, Blaszczyk-Lezak I, Griol A, Barrios CA, et al. Adv Mater 2011;23:761.

[5] Lee HM, Kim YN, Kim BH, Kim SO, Cho SO. Adv Mater 2008;20:2094-8.

[6] Duan H, Winston D, Yang JKW, Cord BM, Manfrinato VR, Berggren KK. J Vac Sci Technol B 2010;28:C6-58.
[7] Wang S, Pile DFP, Sun C, Zhang X. Nano Lett 2007;7:1076.

[8] Teh WH, Smith CG. J Vac Sci Technol B 2003;21:3007.

[9] Teh WH, Liang C-T, Graham M, Smith CG. I Microelectromech Syst 2003; $12: 641$.

[10] Badmaeva IA, Surovtsev NV, Malinovskii VK, Sveshnikova LL. J Struct Chem 2010;51:244. 